\title{
Partially Wetting Thin Liquid Films: Structure and Dynamics Studied with Coherent X Rays
}

\author{
C. Gutt, ${ }^{1,2, *}$ M. Sprung, ${ }^{3}$ R. Fendt, ${ }^{1}$ A. Madsen, ${ }^{4}$ S. K. Sinha,${ }^{2}$ and M. Tolan ${ }^{1}$ \\ ${ }^{1}$ Experimentelle Physik I, Universität Dortmund, Otto-Hahn Straße 4, D-44221 Dortmund \\ ${ }^{2}$ Department of Physics, University of California at San Diego, La Jolla, California 92093, USA \\ ${ }^{3}$ Argonne National Laboratory, 9700 South Cass Avenue, Building 432 E003, Argonne, Illinois 60439, USA \\ ${ }^{4}$ European Synchrotron Radiation Facility, BP 220, 38043 Grenoble Cedex, France
}

(Received 24 April 2007; published 31 August 2007)

\begin{abstract}
We studied the surface structure of thin liquid films vapor deposited on solid substrates in a partial wetting situation by means of coherent $x$-ray scattering. No dynamics has been observed showing the absence of capillary waves on liquid films partially wetting a substrate. Instead an exponential form of the height-height correlation function has been found pointing toward a solidlike behavior of the thin liquid films at large length scales. The exact surface structure and degree of replication with the substrate depend on the deposition rate of the molecules.
\end{abstract}

DOI: 10.1103/PhysRevLett.99.096104

PACS numbers: 68.08.Bc, 68.15.+e

Solid substrates exposed to a vapor atmosphere are omnipresent in everyday life. The phenomena of adsorption and wetting of fluids on solid substrates have been a long-standing topic of fundamental research and technological interest since they determine, for example, the homogeneity of thin films and coatings. However, wetting is a complex phenomenon, as it is highly sensitive to roughness, chemical potentials, and chemical contaminants [1]. A quantity reflecting the underlying interaction potentials is the time dependent height-height correlation function $C(R, t)=\langle\xi(r, t) \xi(r+R, t)\rangle$ of the surface contour $\xi(r, t)$ of a fluid film.

In the case of complete wetting and van der Waals interactions the situation is rather simple within the Deryagin approximation. The van der Waals interaction between solid and liquid determines not only the equilibrium thickness but also the surface structure of the film $[2,3]$. The Fourier transform or power spectral density (PSD) $\tilde{C}(q, t)$ of the real space correlation function of the wetting film can be written as

$$
\tilde{C}(q, t)=\chi^{2}(q) \tilde{C}_{\mathrm{sub}}(q)+\tilde{C}_{\mathrm{int}}(q, t),
$$

where $\tilde{C}_{\text {sub }}(q)$ denotes the PSD of the underlying solid substrate which is transferred to the fluid layer. The factor $\chi(q)$ determines to which extent the substrate contour is replicated in the surface contour of the fluid film. In the Deryagin approximation the replication factor is of Lorenzian form with a characteristic healing length $\zeta$ depending on the surface tension of the liquid and the substrate-film interaction. Besides the replicated Fourier components from the substrate a fluid film also exhibits an intrinsic dynamic structure due to capillary waves. The corresponding PSD can be written as $\tilde{C}_{\text {int }}(q, t)=$ $\left[\left(k_{B} T\right) / \gamma\right]\left[l^{4} /\left(q^{2} l^{4}+\zeta^{2}\right)\right] f(q, t)$ where the function $f(q, t)$ describes the time dependence of the capillary waves. Surface sensitive $\mathrm{x}$-ray scattering experiments have shown that the static average of Eq. (1) is a valid description for vapor deposited films in a complete wetting situation [4,5]. Experiments using surface sensitive x-ray photon correlation spectroscopy have measured the dynamic structure factor $f(q, t)$ of thin polymer films completely wetting a silicon substrate [6]. The results have been found to be in good agreement with capillary wave theory.

In contrast to complete wetting the situation of partial wetting and dewetting is more complex and has been mostly addressed for thin polymer films. Here a large body of work, mainly done by atomic force microscopy, was performed concerning the structure which forms during dewetting situations (see, e.g., [7-9], and references therein). In this Letter we present the first study of the surface structure of vapor deposited thin liquid films in a partial wetting situation. The use of coherent $x$ rays in this study enabled us to follow the exact spatial arrangement of the film surface and quantify an eventual dynamic behavior.

Two highly volatile liquids, $n$-hexane and cyclohexane with van der Waals diameters around $6 \AA$, have been vapor deposited on silicon and glass substrates. The substrates have been cleaned in strong oxidizing solutions with subsequent rinsing of water. The remaining film was blown off with dry nitrogen gas. For the film deposition a glass reservoir was filled with the desired liquid, evacuated, and then connected to the sample. The sample cell was evacuated to pressures below $10^{-4}$ mbar and the vapor was led into the cell by opening the valve for a short period of time. The liquid molecules condense on the substrate by slightly lowering the substrate temperature by about $4-8{ }^{\circ} \mathrm{C}$. This cooling can be performed rapidly within minutes or very slowly within, e.g., $24 \mathrm{~h}$. Once a film thickness has been prepared the temperature is kept constant during the x-ray measurement. No bulk phase was present in the system and the films have been grown in an undersaturated gas atmosphere. By contrast, previous x-ray scattering experiments of thin wetting films have been performed under saturated conditions [4,5]. It has been 
observed before for thin liquid helium films that the surface structure depends on the atmosphere being saturated or undersaturated [10]. The thickness of the films can be controlled by changing the temperature of the substrate. $\mathrm{X}$-ray reflectivity measurements allowed us to study the thickness and stability of the thin films. Film thicknesses from monolayer coverage up to $80 \mathrm{~nm}$ have been investigated.

The results reported have been obtained in several experimental runs using coherent $\mathrm{x}$-ray scattering at beam lines ID10A (ESRF) and 8ID (APS). The experimental setups used were similar to those reported in Refs. [6,11]. At the APS an x-ray energy of $7.4 \mathrm{keV}$ was employed and a partially coherent beam was produced by a $20 \mu \mathrm{m}$ pinhole in front of the sample. A direct illumination CCD camera was located $3450 \mathrm{~mm}$ downstream of the sample. The angle between the $\mathrm{x}$-ray beam and the sample surface was $0.1^{\circ}$, which is below the angle of total external reflection resulting in a penetration depth of $75 \AA$. This ensures that the experiment is highly surface sensitive and the measured scattering signal is proportional to the PSD of the liquid surface, i.e., $S(q, t) \propto \tilde{C}(q, t)$. For each film a series of CCD images was taken with typical exposure times varying between 0.3 and $1.8 \mathrm{~s}$ for a length of approximately $20 \mathrm{~min}$. Summing up all CCD images and averaging over pixels with constant $q$ values leads to an ensemble averaged $\langle S(q)\rangle$ with $q=\sqrt{q_{x}^{2}+q_{y}^{2}}$. Figure 1 displays the structure factors for different films. For the thinner films the substrate is within the penetration depth of the x-ray beam. However, the substrates used were smooth (rms roughness $\sigma \approx 2-3 \AA$ ) resulting in a weak diffuse scattering signal only. Even the 60 and $100 \AA$ thick films produced a diffuse scattering signal which is already a factor 3-5 times larger than the empty substrate, pointing towards a rather large surface roughness of the thin liquid

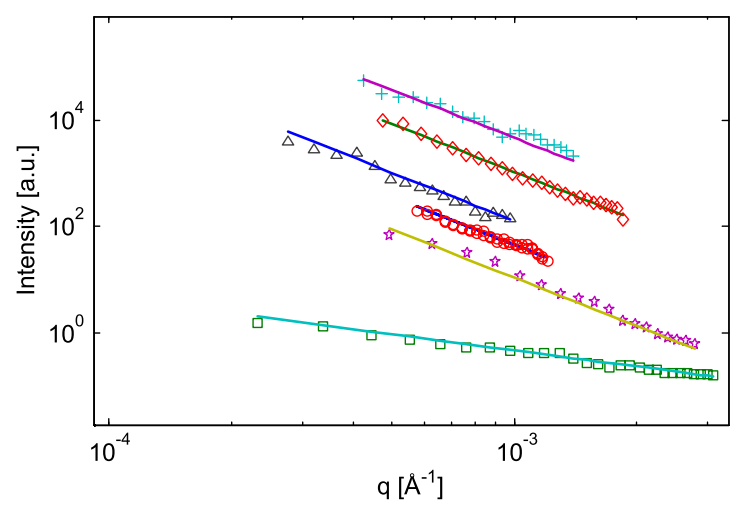

FIG. 1 (color online). $\quad S(q)$ from vapor deposited thin liquid films adsorbed on silicon and glass substrates. The film thicknesses are from top to bottom $600,500,400,300,100$, and $60 \AA$. The 300 and $400 \AA$ thick films correspond to cyclohexane films adsorbed on glass substrate, while all other data correspond to hexane films on silicon substrates. The $60 \AA$ film shows a $S(q) \propto$ $q^{-1}$ behavior. All thicker films can be described by $S(q) \propto q^{-3}$. films. Because of this observation of a drastically enhanced scattering signal by condensing the liquid film on the substrate we assume the scattering signal to be dominated by the liquid film rather than the substrate. The bottom line represents the scattering from a $60 \AA$ thick hexane film on a silicon substrate which shows a $\langle S(q)\rangle \propto q^{-1}$ behavior. The same $q$ dependence has been observed for the empty silicon wafers implying that the surface contour of the wafer is partially transferred to the thin liquid film. Such a behavior would be in agreement with the predictions for complete wetting. However, for thicker films a different behavior can be observed. The other data points in Fig. 1 represent selected examples of scattering from hexane and cylcohexane films with a thickness between 100 and $600 \AA$ adsorbed on silicon and glass substrates. All measured data sets of films with a thickness larger than $100 \AA$ can be fitted with a power law behavior according to $\langle S(q)\rangle \propto q^{-3}$. This form of the surface PSD deviates from films decorated by capillary waves and is an unambiguous sign for films in a nonperfect wetting situation. The same peculiar PSD has been observed for thin polymer films in the early stage of dewetting [12]. In contrast to the logarithmic decaying real space correlation function for capillary waves the observed $q^{-3}$ behavior suggests a self-affine fractal surface structure with a universal scaling exponent $H=0.5$ for the liquid films $[13,14]$. Such surface structures have been observed for many solid surfaces including vapor deposited solid films on solid substrates; see, e.g., [15,16]. Interestingly, the corrugated surface structure of liquid films in a partial wetting situation also falls into this category of surface structures. The corresponding real space height-height correlation function is of exponential form with an in-plane correlation length $\xi$ [17] $C(R)=\sigma^{2}(\Gamma, l) \exp (-R / \xi)$ where the rms roughness $\sigma$ depends on the deposition rate $\Gamma$ and the film thickness $l$. With this correlation function the corresponding intrinsic roughness spectrum of the vapor deposited film takes the form

$$
\tilde{C}_{\text {int }}(q) \propto \frac{\sigma^{2} \xi^{2}}{\left(1+q^{2} \xi^{2}\right)^{3 / 2}},
$$

which yields the correct asymptotic form if $q \xi \gg 1$. From the data we estimate $\xi \gg 3000 \AA$ as the lower limit of the in-plane correlation length.

The $q^{-1}$ dependence for small film thicknesses implies the existence of a crossover region from $\tilde{C}(q) \propto \tilde{C}_{\text {sub }}$ to $\tilde{C}(q) \propto \tilde{C}_{\text {int }}$ as a function of film thickness. This suggests in analogy to Eq. (1) a film thickness dependent replication factor $\chi(q, l)^{2} \tilde{C}_{\text {sub }}$ also for partial wetting films.

In order to investigate the question of film replication at small $q$ values and for thin films in more detail we performed in situ rocking scans of the sample during vapor deposition. Figure 2 displays such rocking curves of thin hexane films recorded at $q_{z}=5.8 \times 10^{-2} \AA^{-1}$. The data in Fig. 2(a) represent scattering from the empty substrate, the substrate with monolayer coverage, and films of 30,60, 100, and $140 \AA$ thickness, respectively. The films have 

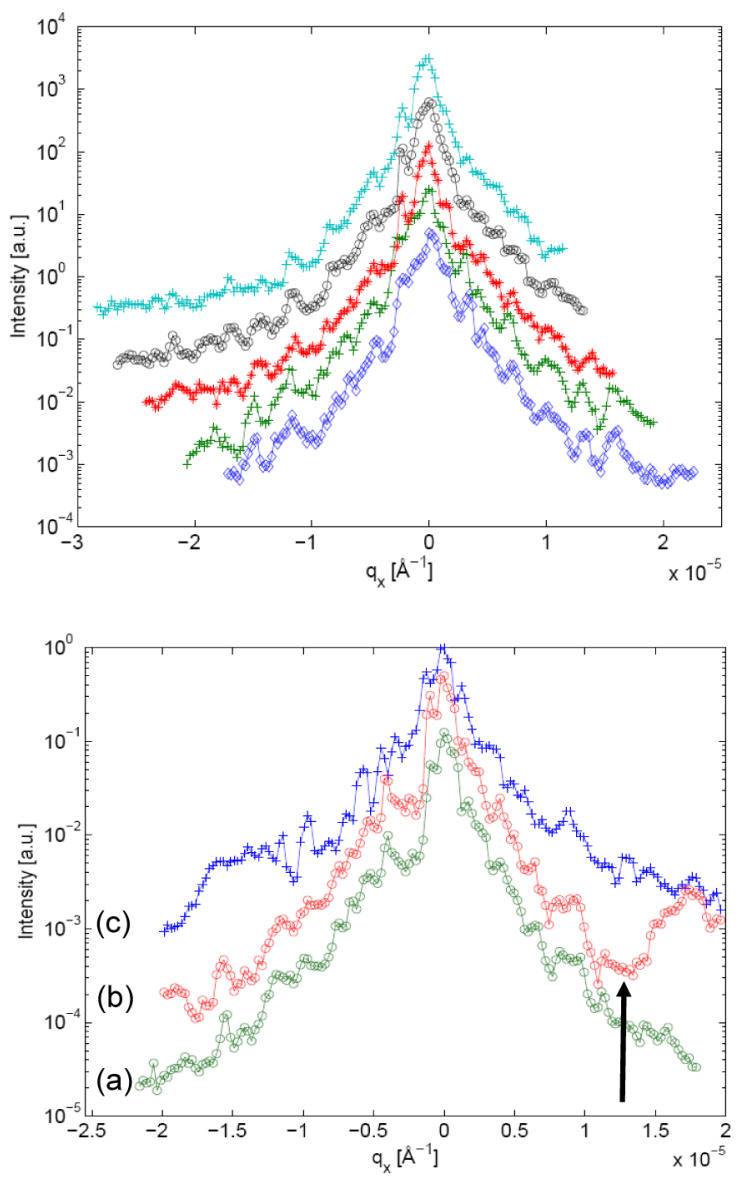

FIG. 2 (color online). Rocking scans of thin liquid vapor deposited hexane films on silicon substrates $\left(q_{z}=5.8 \times\right.$ $10^{-2} \AA^{-1}$ ). (Top) slowly grown films; from bottom to top: monolayer coverage, 30, 60, 100, and $140 \AA$ thick films. (Bottom) rapidly grown films: (a) $30 \AA$ thick film (b) repeated scan, the arrow indicates rapid condensation, (c) $150 \AA$ thick film.

been prepared by slowly cooling the substrate temperature from 24 to $17^{\circ} \mathrm{C}$ within $24 \mathrm{~h}$. All curves show a speckle structure typical for the use of coherent $\mathrm{x}$ rays which is reflecting the exact spatial arrangement without ensemble averaging of the surface structure of both substrate and liquid film. A comparison of the speckle structures yields direct information about the transfer of surface structures from the substrate to the liquid film. Here we see that in the case of slow condensation the speckle structures of the liquid films are similar while not absolutely identical to the one of the underlying substrate implying that the surface structure of the liquid film is following the substrate even in a situation of partial wetting. The degree of replication, however, depends on the deposition rate of the molecules on the substrate. Figure 2(b) displays the corresponding speckle structures for rapid condensation (i.e., $120 \AA$ within 1-2 minutes). Curve (a) represents the speckle structure from a $30 \AA$ thick film. Curve (b) is the repeated scan with a sudden decrease of the substrate temperature indicated by the position of the arrow. The resulting fast condensation of molecules immediately leads to a different surface structure as can be seen by the speckle structure. The surface structure of the final $150 \AA$ thick film (curve (c)) is completely different from the initial substrate surface. Repeated scans showed exactly the same speckle structure. The liquids used are highly volatile liquids with low viscosity which makes it not plausible to expect relaxation times larger than 20 minutes.

As seen before the replication vanishes at larger $q$-values and for thicker films. An eventual surface dynamics of the thin films in the $q$-region where the PSD is of the form $\tilde{C}(q) \propto q^{-3}$ can be studied by investigating the time dependence of the scattered intensity from the CCD images. Figure 3 shows the average of 1000 CCD images with exposure times of $0.5 \mathrm{~s}$ taken from a $400 \AA$ thick hexane film on a silicon substrate. Again a static speckle structure is visible indicating that the film displays no surface dynamics. It is worth mentioning that the van der Waals cutoff of a perfectly wetting thin liquid film of this thickness would be around $1 \times 10^{-5} \AA^{-1}$, i.e., well below the $q$ range covered in the measurement. Also the calculated intensity autocorrelation functions showed no relaxation in the time interval of $1 \mathrm{~s}$ to $20 \mathrm{~min}$. For reasons of comparison, the expected time scales for capillary waves at those length scales are around $10^{-4} \mathrm{~s}$ as calculated from the Navier-Stokes equation with nonslip boundary conditions and a background potential with an effective Hamaker constant [18]. The static speckle structure of the surface PSD becomes even more apparent in Fig. 4, which shows the speckle structure of a $400 \AA$ thick film and from the empty substrate as obtained by a cut along the specular rod (i.e., $q_{y}=0 \AA^{-1}$ ) of one of the averaged CCD images. The signal from the liquid film is more than 1 order of magnitude larger than the empty substrate signal; thus, the CCD images predominantly show the signal from the surface of the thin adsorbed films. The inset shows the speckle structure in more detail. Note that the error bars are smaller than the data points. Such static speckle patterns have been observed for all investigating films. Thus on the addressed length scales the surface is rough but frozen in.

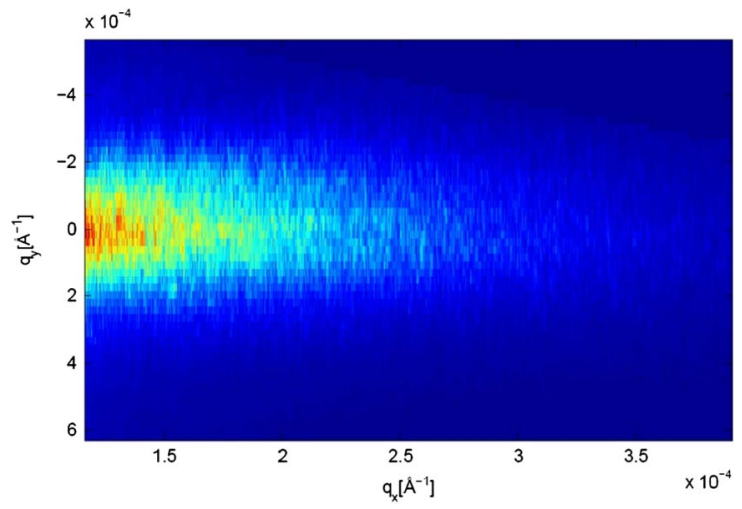

FIG. 3 (color online). CCD images from the scattering of a $400 \AA$ thick hexane film adsorbed on a silicon substrate. 


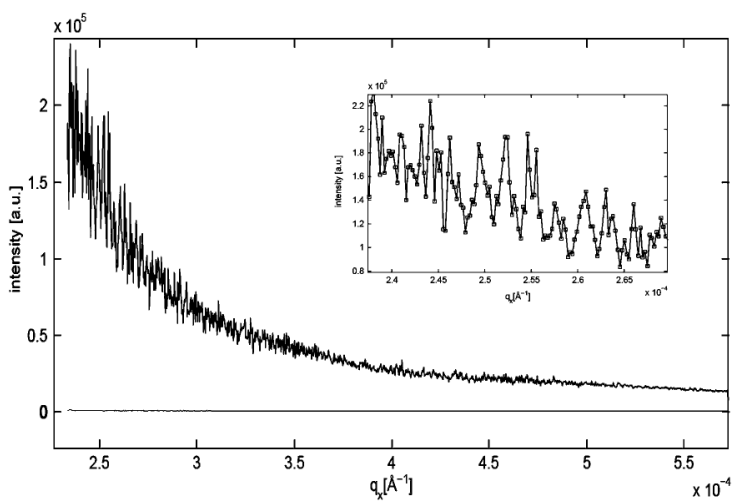

FIG. 4. Speckle signal from a $400 \AA$ thick hexane film on silicon and from the empty substrate as obtained by a cut through the CCD image at $q_{y}=0 \AA^{-1}$. The inset shows the speckle structure in more detail.

For quantifying the degree of surface covering we also performed $\mathrm{x}$-ray reflectivity scans for each film yielding information about the vertical electron density profile of the system substrate-thin liquid film [19]. All profiles obtained show fluctuations in the electron density around the bulk value of the adsorbed liquids and a reduced electron density near the solid-liquid interface; the latter has been observed before for films in a wetting situation [20]. As the averaged electron density for all films found is always close (within a few percent) to the bulk value of the liquid we exclude large areas of dry patches on the surface.

In summary, the surface structure of thin vapor deposited liquid films in a nonperfect wetting situation proves to be quite complex. We observed static surface structures on length scales ranging from $10^{6}$ to $10^{3} \AA$.

In the case of $n$ hexane the experiments have been performed at temperatures far above the freezing point of bulk hexane $\left(-95.6{ }^{\circ} \mathrm{C}\right)$. The bulk freezing point of cyclohexane $\left(6.5^{\circ} \mathrm{C}\right)$ is closer to the experimental conditions; however, the similarities observed between the hexane and the cylcohexane films point toward a different mechanism than solidification through freezing. Capillary waves are a long ranging, collective motion of molecules on length scales ranging from $\mathrm{cm}$ to $\mathrm{nm}$. Partial wetting leads to a nonuniform film which causes the classical hydrodynamics as expressed by the Navier-Stokes equation with proper boundary conditions no longer to be valid on the probed length scales. The result is that the films behave in a solid manner on these length scales. The nonuniformity of the film is visible in the diffuse scattering with a peculiar form of the PSD $\propto q^{-3}$ which is usually not seen on liquid surfaces and is indicating a self-affine fractal surface structure with a rather long correlation length $\xi \gg 3000 \AA$. Moreover, a second length scale exists in the system which is a measure of the substrate-adsorbate interaction and is therefore in close analogy to the van der Waals cutoff present in complete wetting films. This length scale is visible via the transition of the PSD from a $q^{-1}$ to a $q^{-3}$ powerlaw by increasing the film thickness. Below this length scale the influence of the substrate-adsorbate interaction is small and the liquid surface does not follow the substrate surface but rather shows the $q^{-3}$ power law in the scattering signal. At larger length scales the liquid surface follows the substrate contour if the adsorption rate is small. At high adsorption rates a surface structure forms which does not follow the substrate with the relaxation process towards equilibrium hindered by the partial wetting situation.

*Present address: Hasylab/DESY, Notkestrasse 85, D22607 Hamburg, Germany.

[1] P. G. deGennes, Rev. Mod. Phys. 57, 827 (1985).

[2] D. Andelman, J.-F. Joanny, and M. O. Robbins, Europhys. Lett. 7, 731 (1988).

[3] M. O. Robbins, D. Andelman, and J.-F. Joanny, Phys. Rev. A 43, 4344 (1991).

[4] I. M. Tidswell, T. A. Rabedeau, P. S. Pershan, and S. D. Kosowsky, Phys. Rev. Lett. 66, 2108 (1991).

[5] A. K. Doerr, M. Tolan, W. Prange, J.-P. Schlomka, T. Seydel, W. Press, D. Smilgies, and B. Struth, Phys. Rev. Lett. 83, 3470 (1999).

[6] H. Kim, A. Rühm, L. B. Lurio, J. K. Basu, J. Lal, D. Lumma, S. G. J. Mochrie, and S. K. Sinha, Phys. Rev. Lett. 90, 068302 (2003).

[7] R. Seemann, S. Herminghaus, and K. Jacobs, Phys. Rev. Lett. 86, 5534 (2001).

[8] H. I. Kim, C. Mathew Mate, K. A. Hannibal, and S.S. Perry, Phys. Rev. Lett. 82, 3496 (1999).

[9] R. Konnur, K. Kargupta, and A. Sharma, Phys. Rev. Lett. 84, 931 (2000).

[10] L. B. Lurio, T. A. Rabedeau, P. S. Pershan, I. F. Silvera, M. Deutsch, S. D. Kosowsky, and M. M. Ocko, Phys. Rev. B 48, 9644 (1993).

[11] A. Madsen, T. Seydel, M. Tolan, and G. Grübel, J. Synchrotron Radiat. 12, 786 (2005).

[12] M. Tolan, O. H. Seeck, J.-P. Schlomka, W. Press, J. Wang, S. K. Sinha, Z. Li, M.H. Rafailovich, and J. Sokolov, Phys. Rev. Lett. 81, 2731 (1998).

[13] M. Kardar, G. Parisi, and Y. C. Zhang, Phys. Rev. Lett. 56, 889 (1986).

[14] A.L. Barbasi and H.E. Stanley, Fractal Concepts in Surface Growth (Cambridge University Press, Cambridge, U.K., 1995).

[15] R. Chiarello, V. Panella, J. Krim, and C. Thomson, Phys. Rev. Lett. 67, 3408 (1991).

[16] P. Meakin, Phys. Rep. 235, 189 (1993).

[17] S. K. Sinha, E. B. Sirota, S. Garoff, and H. B. Stanley, Phys. Rev. B 38, 2297 (1988).

[18] J. Jaeckle, J. Phys. Condens. Matter 10, 7121 (1998).

[19] See EPAPS Document No. E-PRLTAO-99-014736 for $\mathrm{X}$-ray reflectivity data. For more information on EPAPS, see http://www.aip.org/pubservs/epaps.html.

[20] A. K. Doerr, M. Tolan, J.-P. Schlomka, and W. Press, Europhys. Lett. 52, 330 (2000). 